Supporting Information

\title{
Catalyst Slurry Preparation Using a Hydrodynamic Cavitation Dispersion Method for Polymer Electrolyte Fuel Cells
}

Hidenori Kuroki, ${ }^{\dagger}, \AA, *$ Keiichiro Onishi, ${ }^{\#}$ Keiichi Asami, ${ }^{\#}$ and Takeo Yamaguchi ${ }^{\dagger}, \star,, * *$

${ }^{\dagger}$ Kanagawa Institute of Industrial Science and Technology (KISTEC), R1-17, 4259 Nagatsuta, Midori-ku, Yokohama, Kanagawa 226-8503, Japan

$\$$ Laboratory for Chemistry and Life Science, Institute of Innovative Research, Tokyo Institute of Technology, R1-17, 4259 Nagatsuta, Midori-ku, Yokohama, Kanagawa 226-8503, Japan

$\S$ Core Research for Evolutionary Science and Technology, Japan Science and Technology Agency (JST-CREST), R1-17, 4259 Nagatsuta, Midori-ku, Yokohama, Kanagawa 226-8503, Japan

\# Nihon Spindle Manufacturing Co., Ltd., 4-2-30 Shioe, Amagasaki, Hyogo 661-8510, Japan

*Corresponding authors: kuroki.h.aa@m.titech.ac.jp (H.K.), yamag@res.titech.ac.jp (T.Y.) 


\section{(A) TEM}

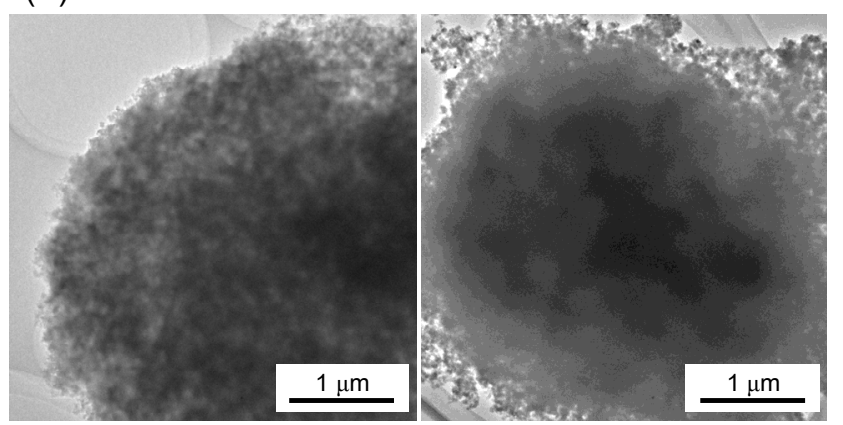

(B) SEM

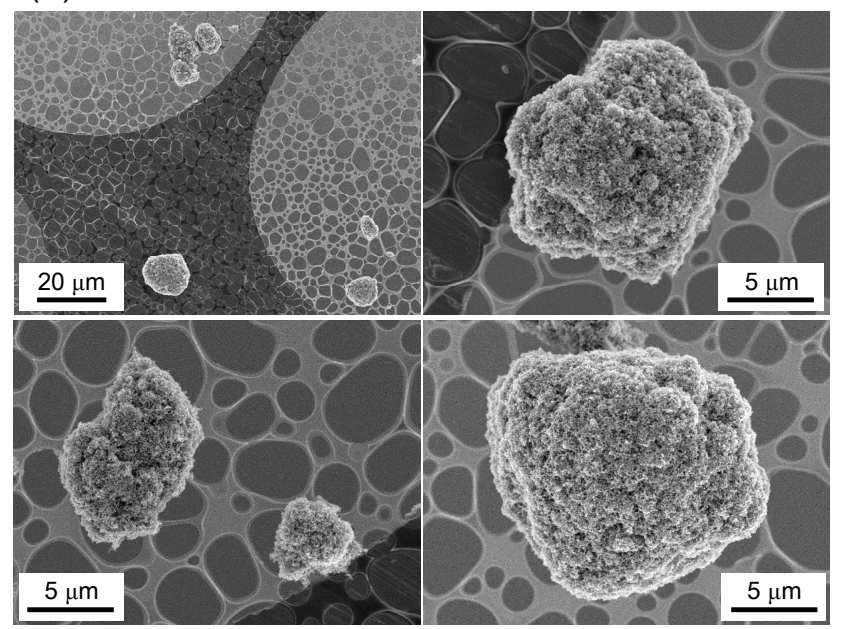

Figure S1. (A) TEM and (B) SEM images of catalyst agglomerates in the untreated Pt/C slurry. 


\section{(A) Sonication}
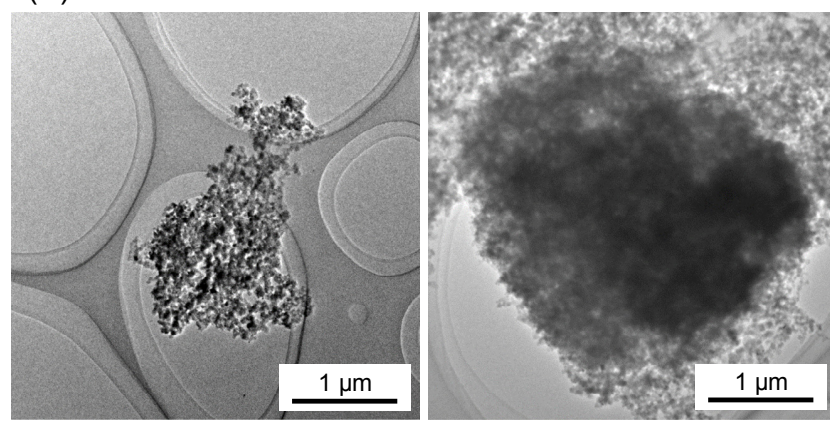

(B) Ball-milling
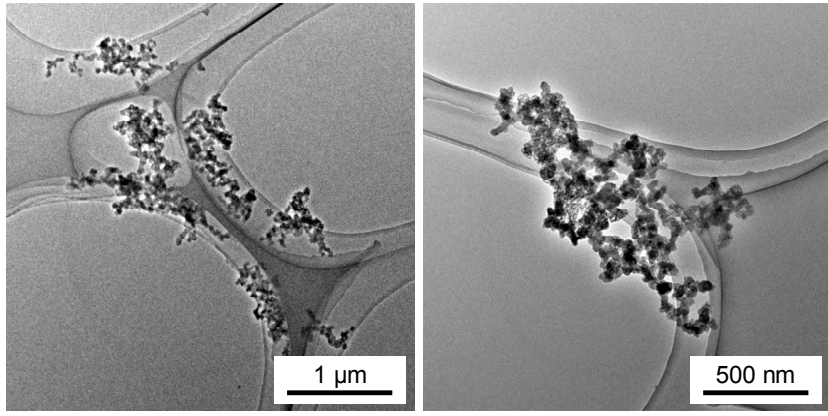

(C) Hydrodynamic cavitation

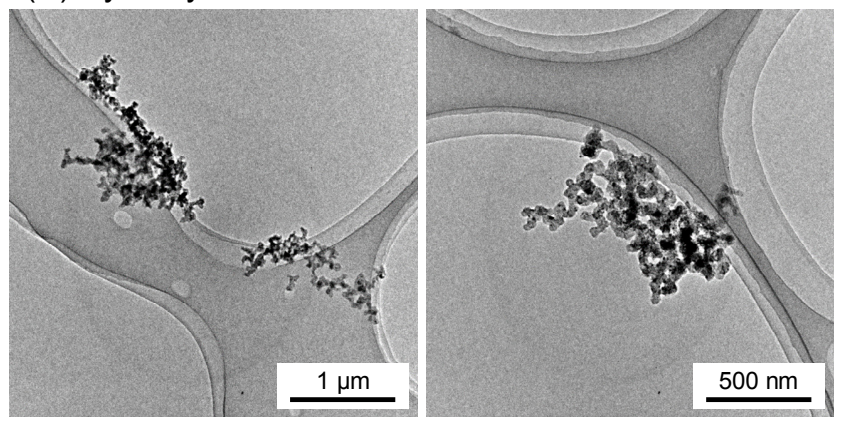

Figure S2. TEM images of the catalyst obtained through the dispersion treatments of (A) sonication, (B) ball-milling, and (C) hydrodynamic cavitation on the $\mathrm{Pt} / \mathrm{C}$ catalyst slurries. 


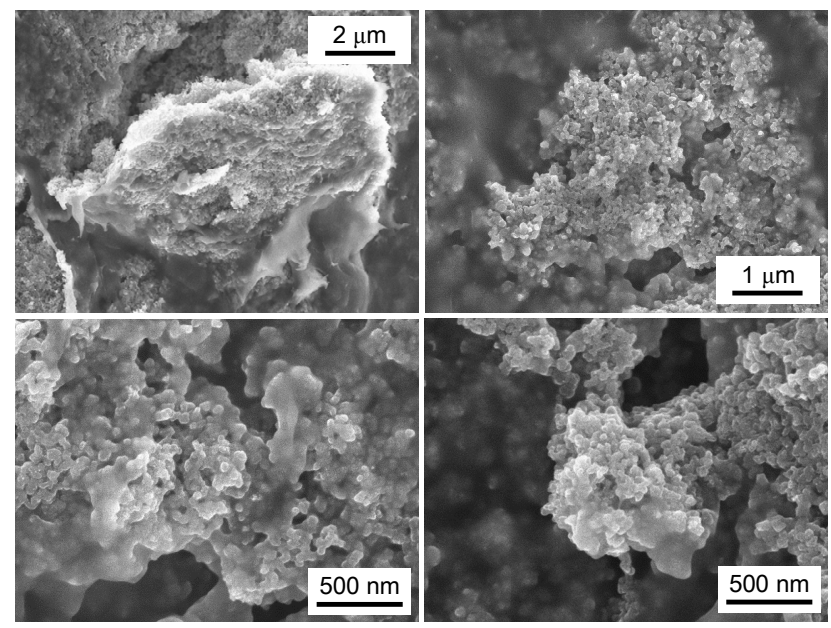

Figure S3. SEM images of the catalyst layer prepared using the untreated $\mathrm{Pt} / \mathrm{C}$ catalyst slurry before the dispersion treatment.

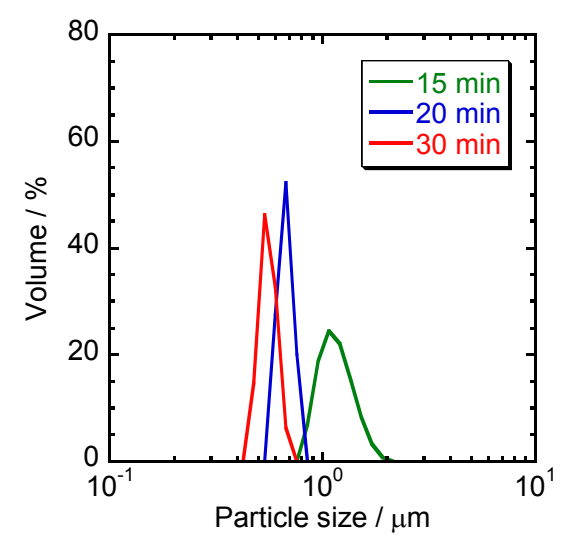

Figure S4. Particle size distribution in the Pt/C catalyst slurries as estimated by DLS analysis. The slurries were prepared via the hydrodynamic cavitation dispersion method with different treatment times. 